\title{
Numerical Analysis of the Unsteady Propeller Performance in the Ship Wake Modified By Different Wake Improvement Devices
}

Tomasz Bugalski, Ph.D.

Ship Design and Research Centre CTO, Poland

Jan A. Szantyr, Prof.

Gdańsk University of Technology, Poland

\begin{abstract}
The paper presents the summary of results of the numerical analysis of the unsteady propeller performance in the non-uniform ship wake modified by the different wake improvement devices. This analysis is performed using the lifting surface program DUNCAN for unsteady propeller analysis. The object of the analysis is a 7000 ton chemical tanker, for which four different types of the wake improvement devices have been designed: two vortex generators, a pre-swirl stator, and a boundary layer alignment device. These produced five different cases of the ship wake structure: the original hull and hull equipped alternatively with four wake improvement devices. Two different propellers were analyzed in these five wake fields, one being the original reference propeller PO and the other - a specially designed, optimized propeller P3. The analyzed parameters were the pictures of unsteady cavitation on propeller blades, harmonics of pressure pulses generated by the cavitating propellers in the selected points and the fluctuating bearing forces on the propeller shaft. Some of the calculated cavitation phenomena were confronted with the experimental. The objective of the calculations was to demonstrate the differences in the calculated unsteady propeller performance resulting from the application of different wake improvement devices. The analysis and discussion of the results, together with the appropriate conclusions, are included in the paper.
\end{abstract}

Keywords: ship wake improvement devices, unsteady propeller performance, numerical analysis

\section{INTRODUCTION}

The research presented in this paper is related to two recent EU projects: PREFUL and STREAMLINE. Within the project PREFUL an extensive modernization of the computer program DUNCAN [1,2] was undertaken. This program is based on the unsteady lifting surface theory and it may be applied to the analysis of unsteady propeller operation in the non-uniform velocity field of the ship's wake. The program DUNCAN can analyse both open and ducted propellers, delivering the time-dependent cavitation extent, unsteady blade and shaft forces and pulsating pressure induced by the cavitating propeller in the prescribed points.

The project STREAMLINE was concerned with the hydrodynamic performance of a 7000 ton chemical tanker $[3,4]$. The model of this ship was equipped with several different flowmodifying devices and tested in a model basin. The tests covered the resistance and propulsion performance of the ship and the unsteady aspects of propeller operation. This paper concentrates on the influence of the wake-improvement devices, such as: vortex generators, pre-swirl stators and boundary layer alignment devices, on the unsteady propeller operation. Altogether five different wake fields are considered in the analysis: the original hull wake, two vortex generators, a pre-swirl stator and a boundary layer alignment device. The objective of the analysis was to determine and compare different aspects of unsteady propeller operation in these wakes, including unsteady cavitation, fluctuating shaft forces and induced pressure pulses. Two propellers were included in the analysis: the reference propeller P0 originally designed for the ship and the propeller P3 as a modified, improved design.

The analysis was performed almost entirely by numerical computations. Only the calculations for the original hull equipped with propeller P0 could be directly compared with the unsteady cavitation photographs and pressure pulses measured on the ship model in a circulating water channel. The results of the analysis are presented in the following sections of this paper. All results given in this paper refer to model scale and the scale of the models is 1:16.5.

\section{WAKE IMPROVEMENT DEVICES AND THEIR EFECT ON THE WAKE STRUCTURE}

The ship considered in this analysis is the 7000 ton chemical tanker with the length between perpendiculars of 94.0 [m], breadth of $15.4[\mathrm{~m}]$, draft of $6.0[\mathrm{~m}]$, block coefficient of 0.786 and the volume displacement of 6826.9 [m3]. The hull geometry is shown in Fig. 1.

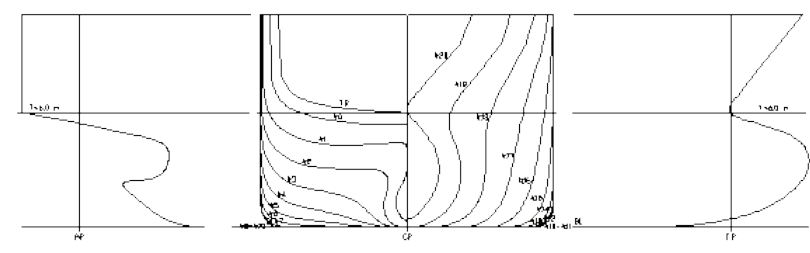

Fig. 1. Geometry of the hull of the chemical tanker 
The photograph of the stern part of the original hull $\mathrm{H} 0$ and the map of the resulting wake velocity field are shown in Fig. 2. The velocity field of the wake seems to be typical for this category of ships and it is reasonably favourable from the point of view of unsteady propeller operation. Consequently, no high intensity of unsteady cavitation phenomena and high induced pressure pulses may be expected.
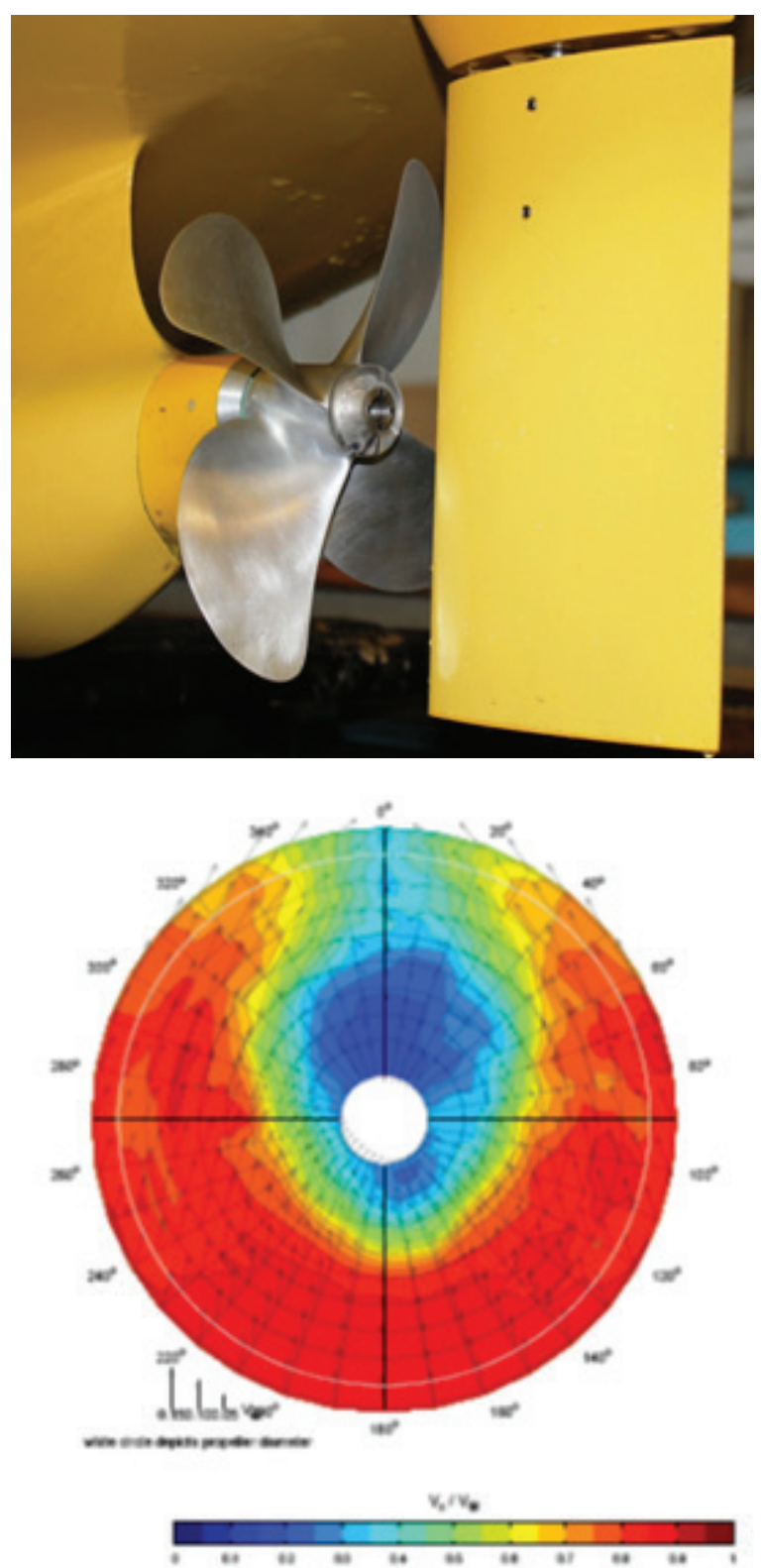

Fig. 2 Photograph of the original hull H0 with propeller $\mathrm{P} 0$ and basic rudder and map of the measured wake velocity field

The vortex generators (VG) were the first tested devices aiming at modification of the wake velocity field. They were fitted both on port and starboard side of the stern in the same positions. The vortex generators VG2 were inclined at an angle of 23 degrees with respect to the waterline. The photograph of the hull equipped with vortex generators VG2 is shown in Fig. 3, together with map of the resulting wake velocity field. The generators have modified the upper part of the wake quite significantly.
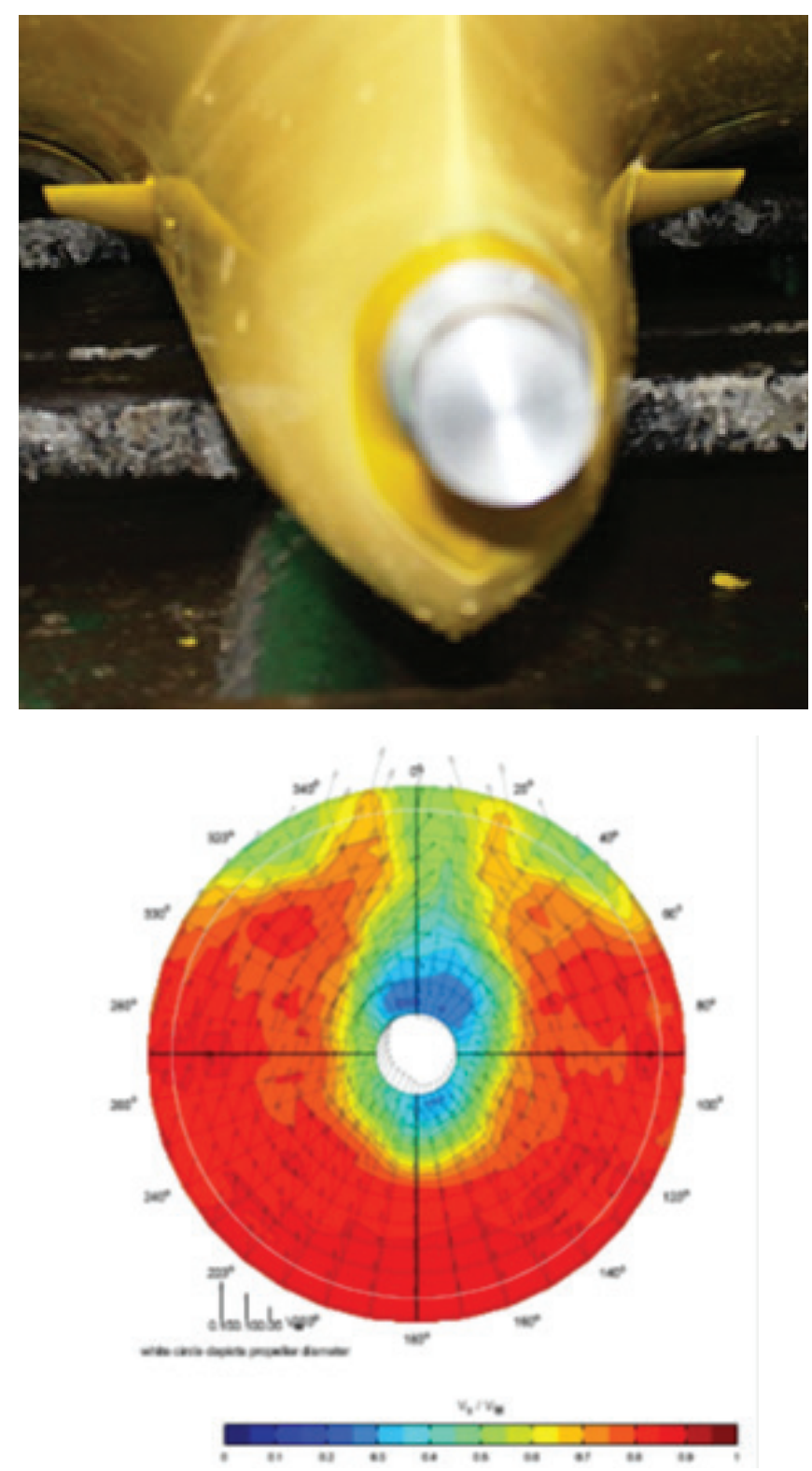

Fig. 3. Photograph of the hull with vortex generators VG2 and map of the measured wake velocity field

The central wake peak was reduced in extent and intensity, particularly in the region right above the shaft, and two additional weak peaks have been added on both sides. These changes should have a positive effect on the unsteady performance of propeller.

The vortex generators VG6 were placed in the same positions as VG2, but they were inclined at an angle of 9 degrees with respect to the waterline. The photograph of the stern with vortex generators VG6 is shown in Fig. 4, together with the map of the resulting wake velocity field. Now the three separate wake peaks visible in the upper part of the wake in Fig. 3 are melted together, creating a wide region of almost uniform velocity. Moreover, the iso-wake lines in this region have an almost circumferential direction. This should have a marked positive effect on the unsteady operation of propellers. 

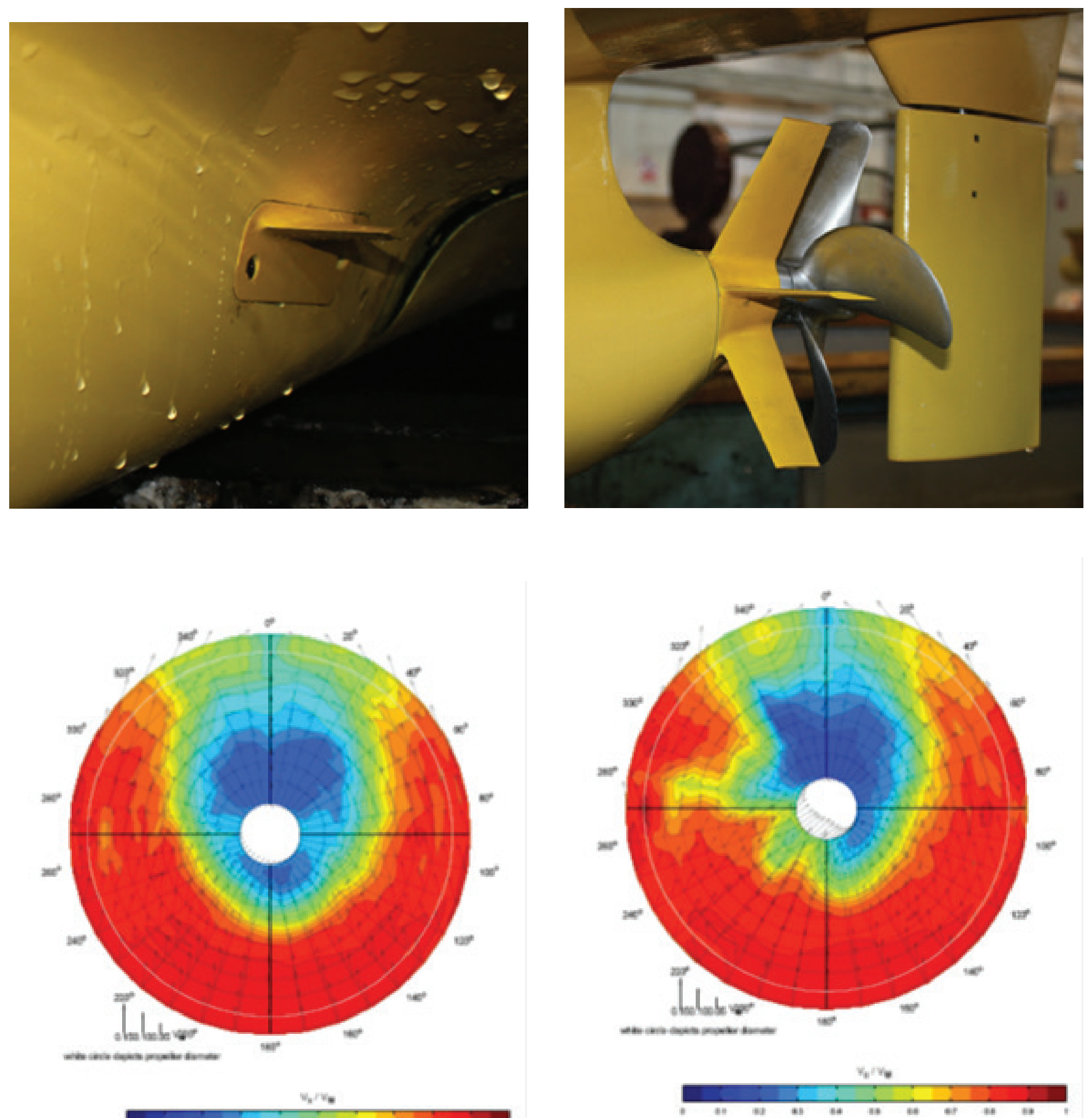

Fig. 5. Photograph of the hull with Pre-swirl Stator PSS and map of the measured wake velocity field

Fig. 4. Photograph of the hull with vortex generators VG6 and map of the measured wake velocity field

Another type of wake improving devices were the PreSwirl Stators (PSS), which were intended first of all to increase the propulsive efficiency. Several configurations of these were tested and finally the one with three stator vanes fitted on the port side of the hull was selected. The photograph of the stern with PSS is shown in Fig. 5, together with the map of the resulting wake velocity field.

Now the main upper peak of the wake is weaker in the top region, but much more intensive and extended towards the starboard side in the region right above the shaft.

At the same time an additional peak has appeared of the port side at approximately 270 degrees position. This should not result in the rise of unsteady cavitation and induced pressure pulses, but it may amplify higher harmonics of the unsteady shaft forces. 
Another type of wake improvement devices was the Boundary Layer Alignment Device (BLAD). It consisted of a couple of spoilers fitted to both sides of the stern part of the hull. Their objective was to prevent thickening of the hull boundary layer in front of the propellers. The photograph of the stern equipped with BLAD is shown in Fig. 6, together with the map of the resulting wake velocity field. The map of the wake does not indicate any decrease on the hull boundary layer thickness. At the same time a quite wide area of retarded flow has appeared in the region right above the shaft. These modifications of the original wake distribution cannot be regarded as positive from the point of view of the unsteady propeller operation.

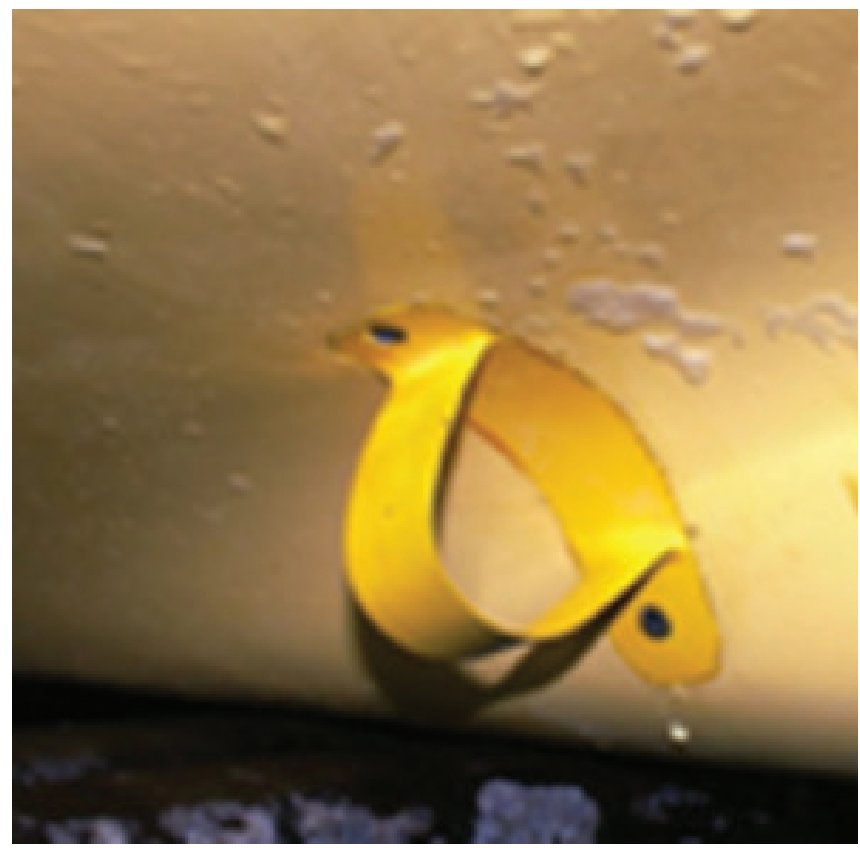

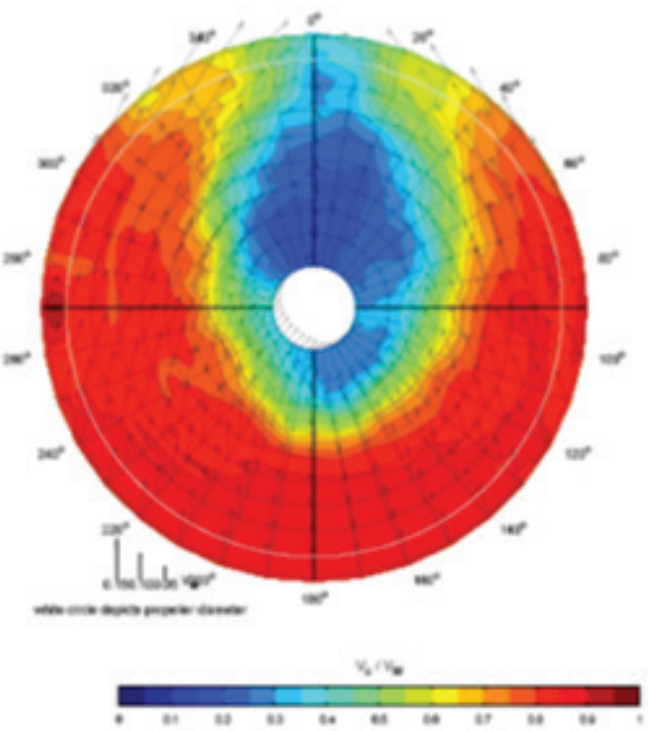

Fig. 6. Photograph of the hull with Boundary Layer Alignment Device BLAD and map of the measured wake velocity field

\section{PROPELLERS INCLUDED IN THE ANALYSIS}

Both propellers considered in the analysis were four bladed and they had the same full scale diameter of $3.8[\mathrm{~m}]$. Their detailed geometry is presented in Figs. 7 and 8. Comparison of both propeller designs shows that the most significant difference between them is the higher hydrodynamic blade tip loading of propeller P3 with respect to the propeller P0. It is visible both in the increased blade pitch and increased mean line camber of the blade sections in this region. This increases the risk of unsteady cavitation. This risk may be further aggravated by a reduction of the expanded blade area ratio from 0.5809 for the propeller P0 to 0.5208 for the propeller $\mathrm{P} 3$ and it may cause an inferior unsteady performance of propeller P3.

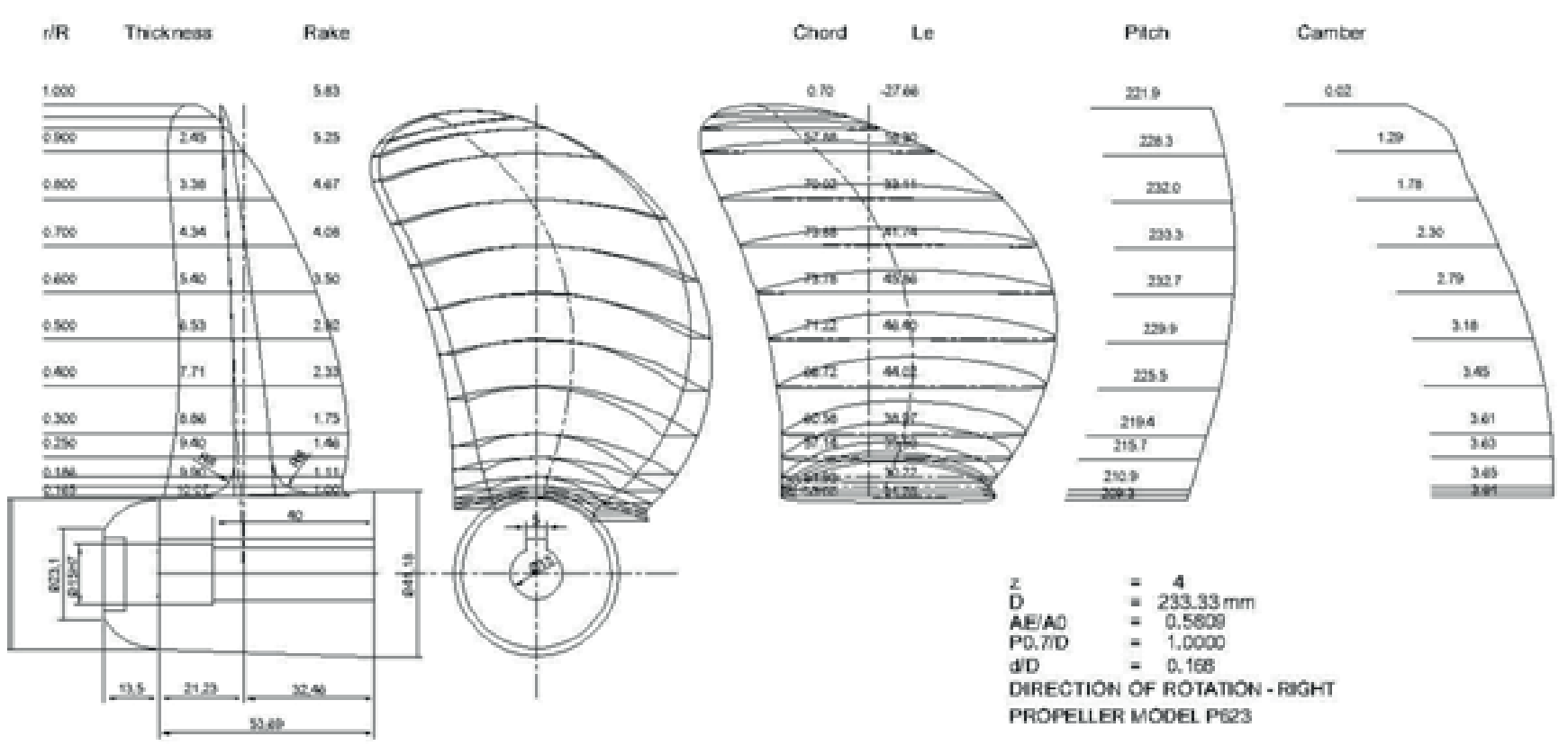

Fig. 7. Drawing of the reference propeller P0 


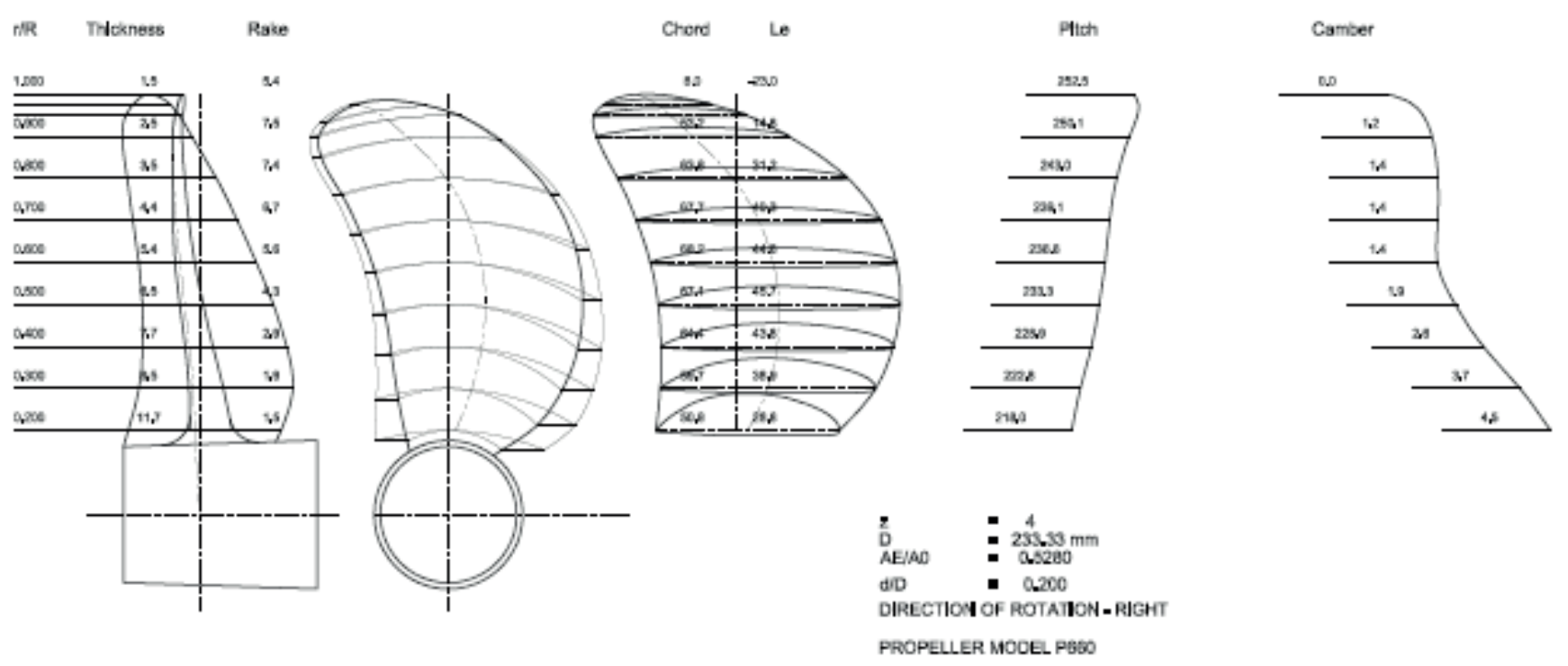

Fig. 8. Drawing of the designed propeller P3

\section{RESULTS OF CALCULATIONS AND COMPARISON WITH EXPERIMENTS}

Calculations reported in this paper were performed using the newly modified computer program DUNCAN. This program is based on the deformable lifting surface theory and it is capable of calculating for the propeller operating in the three-dimensional non-uniform velocity field the timedependent pressure distribution of the propeller blades, the unsteady cavitation extent on the propeller including sheet, bubble and vortex cavitation, the fluctuating bearing forces and the pressure pulses generated by the cavitating propeller in the prescribed points. The program uses a vortex lattice model with 165 bound vortex elements and 180 trailing vortex elements to represent each propeller blade.

Unfortunately, the corresponding experimental results in model scale were available only for the hull $\mathrm{H} 0$ with propeller P0. In these experiments the observations of unsteady cavitation on propeller blades were performed together with the measurements of the pressure pulsations in 17 points on the stern part of the ship hull. The locations of theses points are shown in Fig. 9. As the program allowed for 16 computation points only, and furthermore, the measurements were performed with the rudder in place while the computations did not include the rudder, it was decided to disregard in calculations point no. 1 , located closest to the rudder.

All calculations were performed for the ship speed of 15.0 knots and the cavitation number equal to $\sigma_{\mathrm{N}}=2.632$. This cavitation number is defined in the following way:

$$
\sigma_{N}=\frac{p-p_{V}}{0.5 \rho(n D)^{2}}
$$

where: $\mathrm{p}$ - local static pressure in $[\mathrm{Pa}]$,

$\mathrm{p}_{\mathrm{v}}$ - water boiling pressure in $[\mathrm{Pa}]$,

$\rho$ - water density in $\left[\mathrm{kg} / \mathrm{m}^{3}\right]$,

$\mathrm{n}$ - propeller number of revolutions per second,

D - propeller diameter in metres.
During calculations in the different wake velocity fields the input ship speed was adjusted to produce the same thrust coefficient equal to $\mathrm{K}_{\mathrm{T}}=0.2649$. In this way all the computations of the unsteady parameters of operation of both propellers in all analysed wake fields may be regarded as comparable.

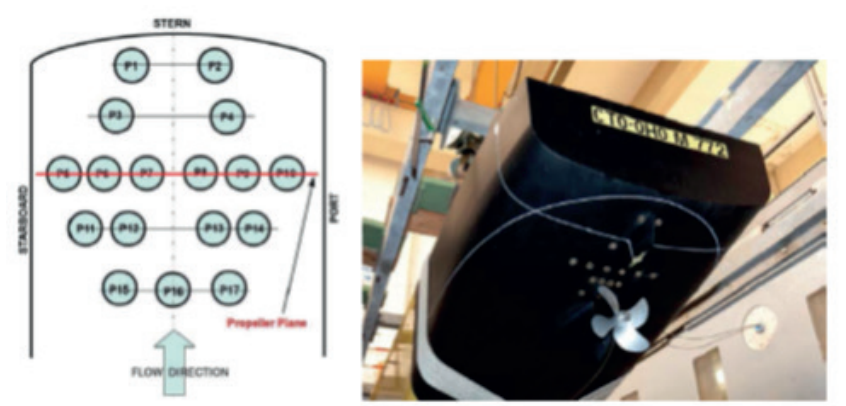

Fig. 9. Location of points for measurement and calculation of the propeller-induced pressure pulses

The observations of cavitation showed some inconsistency in the results, namely in the upper top position of the blade sheet cavitation appeared only occasionally (i.e. in some moments there was no cavitation on the blade in this position, while in the other moments the sheet cavity at the leading edge was clearly visible), leading to a suspicion that there may exist a region of flow separation on the stern part of the hull, generating some unsteadiness of the wake velocity field. Anyway, the calculations indicated a small region of sheet cavitation on the blade in the upper top position, which agrees reasonably well with that observed experimentally at the maximum sheet cavity extent (cf. Fig. 10). 

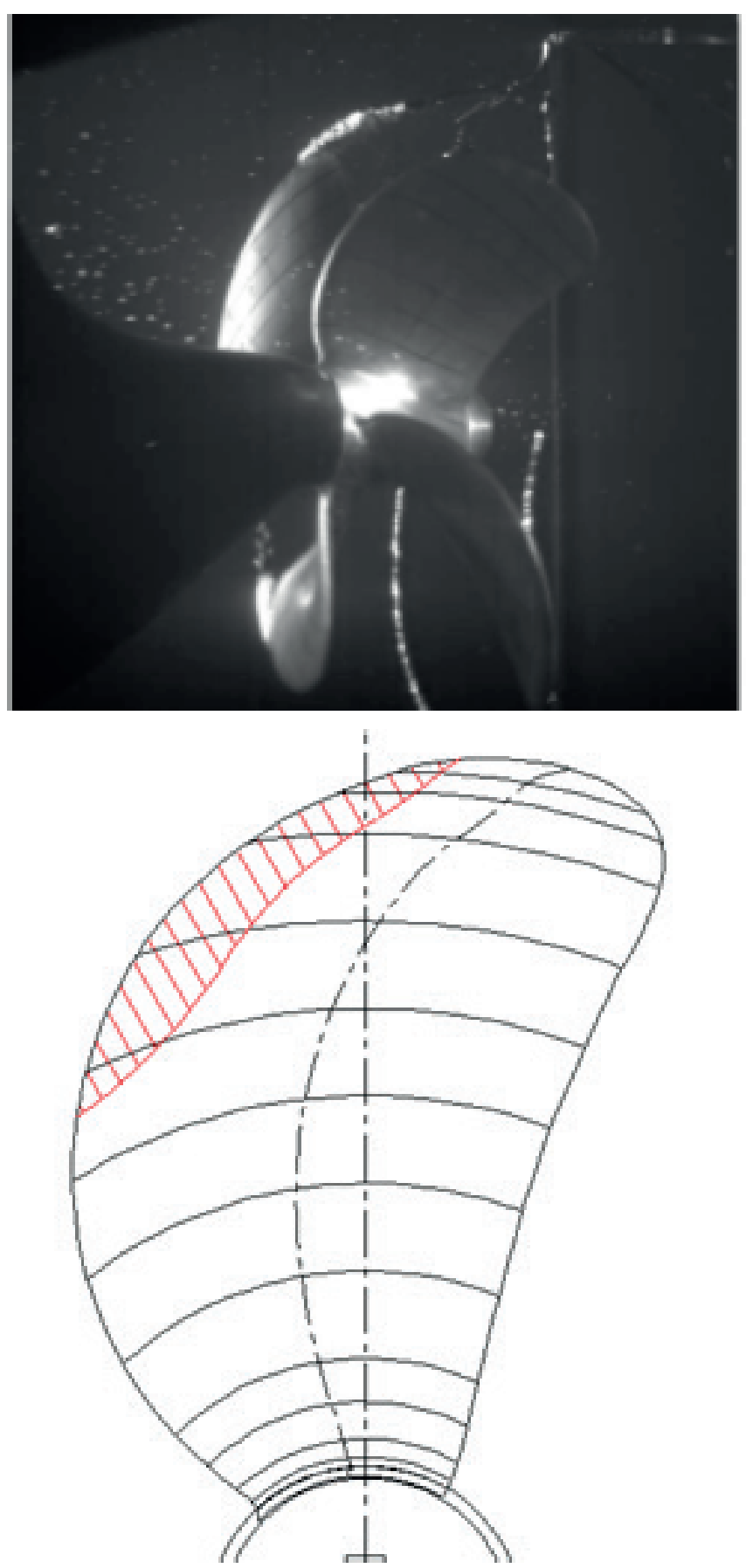

Fig. 10. Comparison of the observed and calculated sheet cavity extent on the blade of propeller $\mathrm{P} 0$ in the vertical position

Fig. 11 shows the comparison of the calculated and experimentally measured blade frequency harmonic amplitudes of the pressure pulses in model scale. The calculated results are visibly higher than the results of corresponding measurements, however both sets of results give values which are well within the acceptable range for this kind of ship. Several reasons for the discrepancy between measurements and calculations may be suggested:

- overestimation of the maximum extent and volume of the pulsating sheet cavity in the calculations, which leads to an overestimation of the maximum amplitudes of the pressure pulses,
- absence of the rudder in the calculations,

- measurements were conducted in the limited space of the circulating water channel, while the calculations assumed unlimited space (except hull surface as the only rigid boundary).

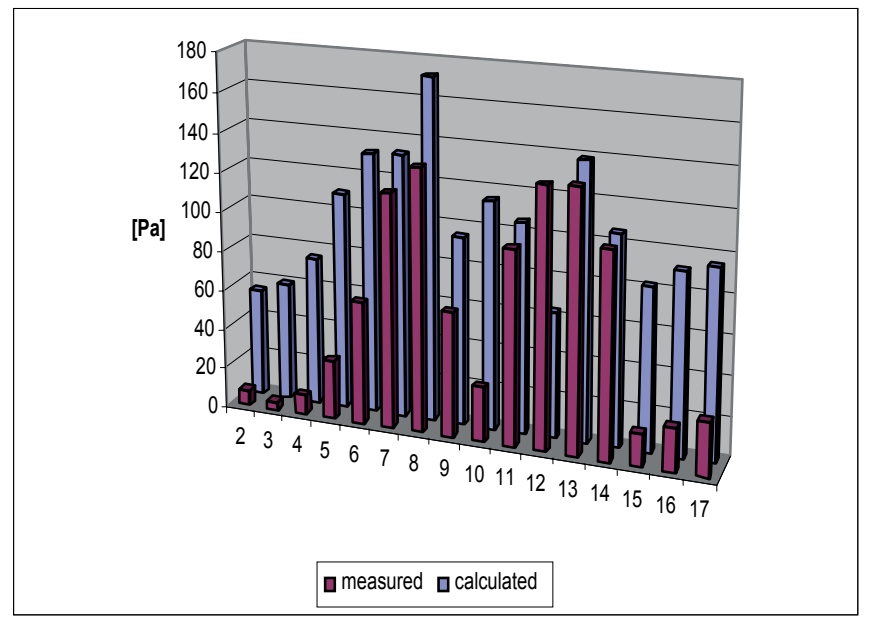

Fig. 11. Comparison of the measured and calculated blade frequency amplitudes of the pressure pulses generated by the propeller $\mathrm{P} 0$ behind the hull $\mathrm{HO}$

The following figures, Figs. 12 to 17, show only the results of calculations. As the unsteady performance of marine propellers is strongly influenced by the cavitation phenomena, Fig. 12 shows the variation of sheet cavity volume, calculated for propeller P0 in model scale for all five wake fields and presented as function of the blade position angle. Blade position angle equal to 0 denotes the upper top position of the blade. The rate of change of the cavity volume with time is the factor primarily responsible for generation of the pressure pulses. The results shown in Fig. 12 may be compared and analysed together with the pressure pulses presented in Fig. 13.

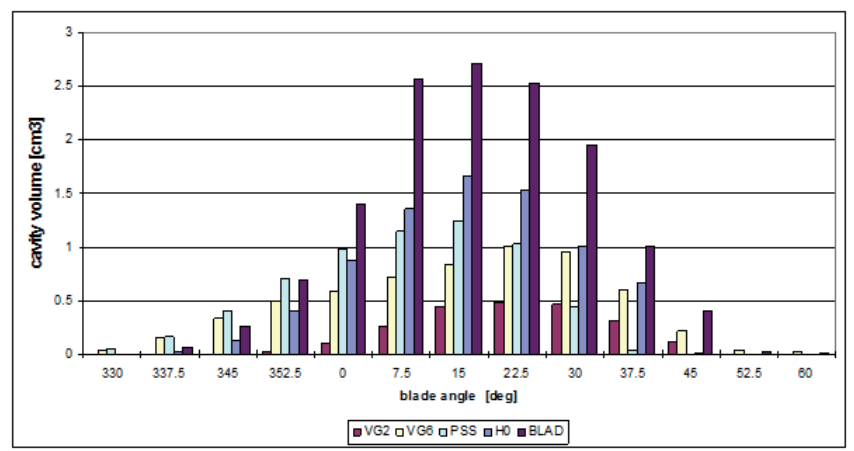

Fig. 12. Calculated cavity volume variation in model scale for propeller P0

The figures 13 and 14 present the comparison of the blade frequency harmonic amplitudes of the pressure pulses and the blade frequency harmonic amplitudes of the bearing forces for the propeller P0 in all analysed wake velocity fields $\mathrm{H} 0$, VG2, VG6, PSS and BLAD. 


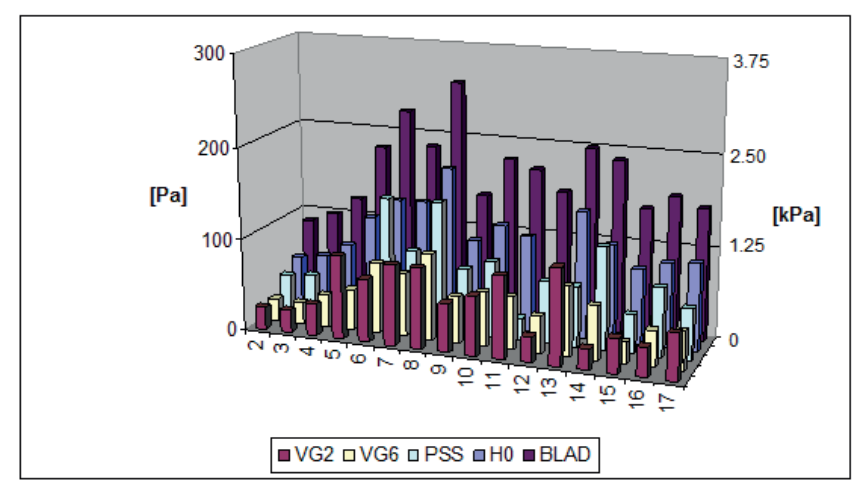

Fig. 13. Comparison of the calculated blade frequency amplitudes of the pressure pulses generated by the propeller $\mathrm{P} 0$ behind the hulls H0, VG2, VG6, PSS and BLAD in model scale ([Pa]) and in full scale $([\mathrm{kPa}])$

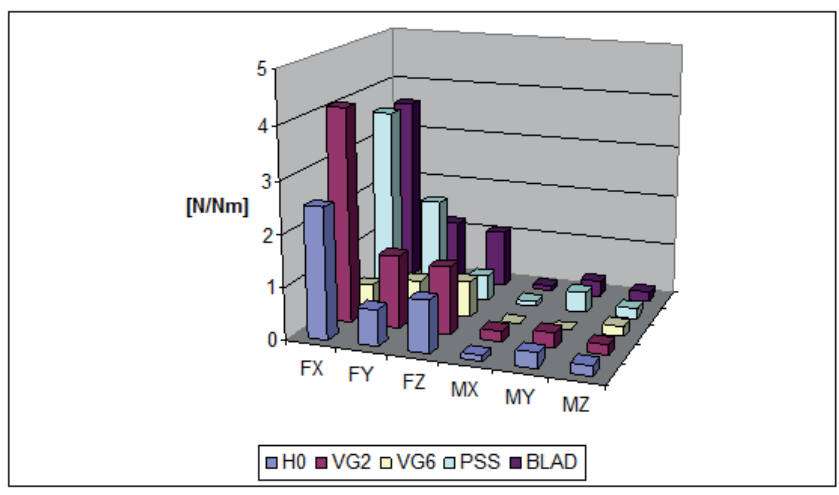

Fig. 14. Comparison of the calculated blade frequency amplitudes of the shaft forces generated on the propeller $\mathrm{P} 0$ behind hulls $\mathrm{H} 0$, VG2, VG6, PSS and BLAD

Fig. 15 shows the variation of sheet cavity volume, calculated for propeller P3 in model scale for all five wake fields and presented as a function of the blade position angle. Similarly as in the case of propeller P0, the results shown in Fig. 15 may be compared and analysed together with the pressure pulses presented in Fig. 16.

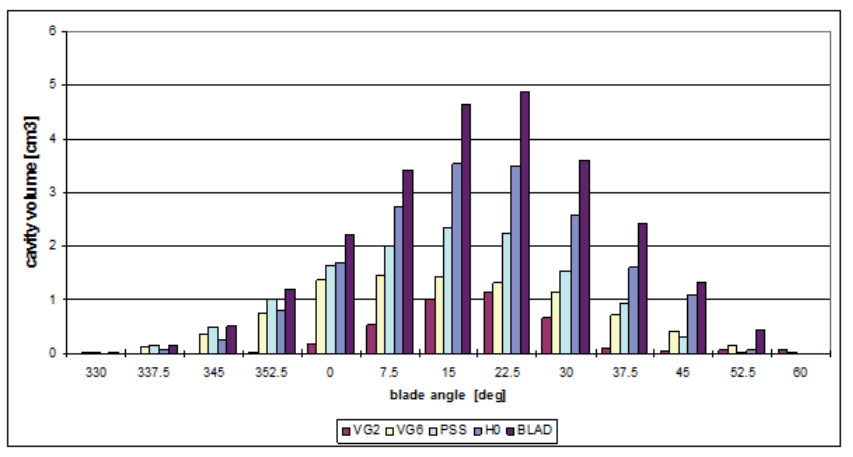

Fig. 15. Calculated cavity volume variation in model scale for propeller P3

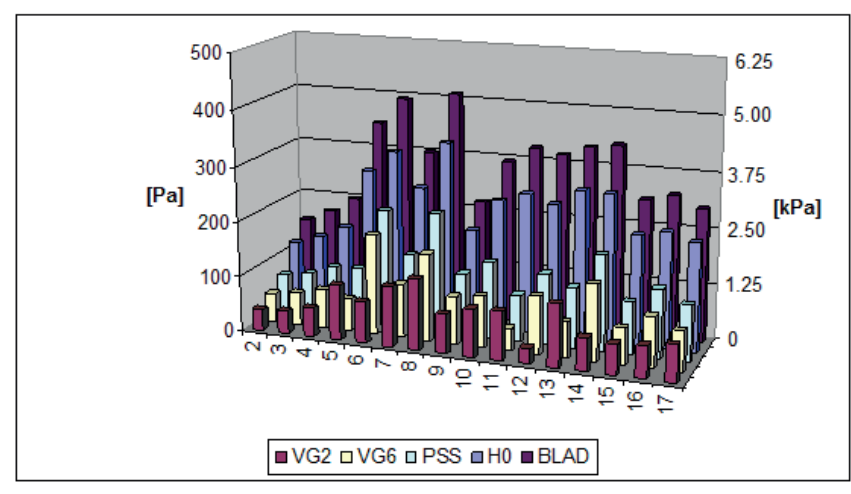

Fig. 16. Comparison of the calculated blade frequency amplitudes of the pressure pulses generated by the propeller P3 behind the hulls H0, VG2, VG6, PSS and BLAD in model scale ([Pa]) and in full scale $([\mathrm{kPa}])$

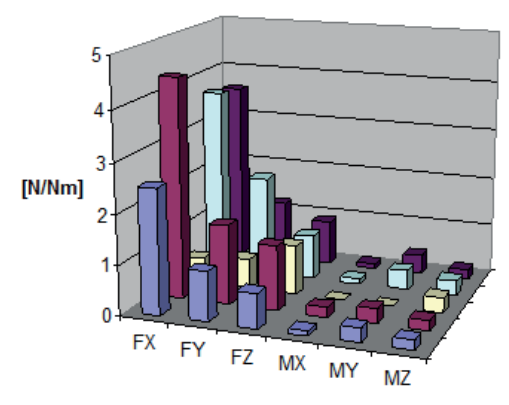

$\square \mathrm{H} 0$ घG2 $\square$ VG6 aPSS $\square \mathrm{BLAD}$

Fig. 17. Comparison of the calculated blade frequency amplitudes of the shaft forces generated on the propeller P3 behind hulls $\mathrm{H} 0$, VG2, VG6, PSS and BLAD

\section{CONCLUSION}

The following conclusions may be drawn from the analysis of the above presented results:

Both computations and experimental observations show very little cavitation on the propeller blades.

The computed blade frequency harmonic amplitudes of the pressure pulses for the hull $\mathrm{H} 0$ and propeller $\mathrm{P} 0$ are visibly higher than the values measured experimentally in the circulating water channel. However, both computed and measured amplitude values are rather low and they would be regarded as acceptable for this type of ship.

The spatial distribution of pressure pulses amplitudes over the stern area in calculations and measurements shows visible differences. The calculated results are much closer to the measured values in the points located near the propeller, while the calculations overestimate the pressure amplitudes more significantly in the regions farther from the propeller. This may result from an overestimation of the maximum size of the pulsating sheet cavity. The pressure signal generated 
by such a cavity fades with distance less rapidly than a signal generated by rotating blades and their variable hydrodynamic loading.

The calculated variation of the sheet cavity volume with blade position showed a strong dependence of this process on the application of respective wake improvement devices. Application of these devices could reduce the cavity volume below the values obtained for the original hull as well as increase it over these values. The best results were achieved for VG2 and the worst for BLAD.

The most effective reduction of the blade frequency harmonic amplitudes of the pressure pulses is achieved with the vortex generators VG2. Next best results are obtained with vortex generators VG6 and the Pre-Swirl Stator. Application of the Boundary Layer Alignment Device BLAD increases the blade frequency harmonic amplitudes.

In all cases the blade frequency harmonic amplitudes of the pressure pulses for the propeller P3 are significantly higher than the corresponding values for the original propeller P0. This follows from a rather unusual geometry of the propeller $\mathrm{P} 3$ as compared to the propeller P0.

The most effective reduction of the blade frequency amplitude of the bearing forces (practically the fluctuation of thrust is a decisive factor here) is achieved with the vortex generator VG6 for both propellers. As far as the fluctuating bearing forces are concerned, both propellers P0 and P3 are fully comparable. The general level of fluctuation of the bearing forces should be regarded as low and fully acceptable in all analysed cases.

Considering all results of calculations presented in this paper the vortex generator VG6 should be considered as the most effective device as far as the unsteady performance of the propeller is concerned.

\section{ACKNOWLEDGEMENTS}

The experimental results presented in this paper have been obtained in STREAMLINE project. The STREAMLINE project is a collaborative $\mathrm{R} \& \mathrm{D}$ project, partly funded by the $7 \mathrm{th}$ Framework Programme of the European Commission. The authors would like to express their gratitude for the possibility of using these results in preparation of this paper.

\section{BIBLIOGRAPHY}

1. Glover E.J., Szantyr J. A.: The Analysis of Unsteady Propeller Cavitation and Hull Surface Pressures for Ducted Propellers, Trans. RINA, Vol. 132, 1990.

2. Szantyr J. A.: User Instructions for the Program DUNCAN2012 for the Analysis of Ducted Propeller Operation in the Non-uniform Velocity Field, Technical Report of CTO SA No RH-2012/T-123 (in Polish), 2012.

3. Di Felice F., Salvatore F., Dymarski P., Pereira F.: Deliverable D21.1 -Results of Model Tests on Reference Configurations. STREAMLINE project Technical report, INSEAN and CTO, 2011.

4. Di Felice F., Falchi M., Aloisio G., Pecoraro A., Salvatore F., Bugalski T.: Deliverable D21.11 -Results of Model Tests on Optimized Configurations. STREAMLINE project Technical report, INSEAN and CTO, 2013.

\section{CONTACT WITH THE AUTHORS}

Tomasz Bugalski, Ph.D.

Ship Design and Research Centre CTO

Szczecińska 65

80-392 Gdańsk,

Poland

email: tomasz.bugalski@cto.gda.pl

Jan Szantyr, Prof.

Gdańsk University of Technology

11/12 Narutowicza Street

80-233 Gdańsk, Poland

email: jas@pg.gda.pl 